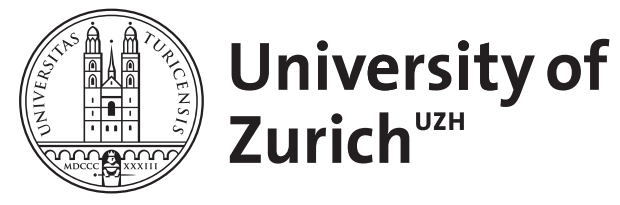

Zurich Open Repository and Archive

University of Zurich

University Library

Strickhofstrasse 39

CH-8057 Zurich

www.zora.uzh.ch

Year: 2012

Coronary steal phenomenon due to ipsilateral upper extremity arteriovenous fistula in a patient with internal thoracic artery bypass graft : An alternative treatment strategy

Musto D’Amore, Sergio ; Zipponi, Manuel ; Hagne, Christian ; Pedrazzini, Giovanni B

Posted at the Zurich Open Repository and Archive, University of Zurich

ZORA URL: https://doi.org/10.5167/uzh-75235

Journal Article

Published Version

Originally published at:

Musto D'Amore, Sergio; Zipponi, Manuel; Hagne, Christian; Pedrazzini, Giovanni B (2012). Coronary steal phenomenon due to ipsilateral upper extremity arteriovenous fistula in a patient with internal thoracic artery bypass graft : An alternative treatment strategy. Cardiovascular Medicine, 15(12):365367. 


\title{
Coronary steal phenomenon due to ipsilateral upper extremity arteriovenous fistula in a patient with internal thoracic artery bypass graft
}

\author{
An alternative treatment strategy \\ Sergio Musto D’Amore, Manuel Zipponi, Christian Hagne, Giovanni B. Pedrazzini \\ Cardiocentro Ticino, Lugano, Switzerland
}

\begin{abstract}
Summary
We report the case of a 69-year old woman with an internal thoracic artery bypass, undergoing chronic dialysis through an ipsilateral upper extremity arteriovenous fistula, who experienced typical angina pectoris at rest, with transitory ST segment elevation and angiographic evidence of a coronary steal phenomenon. For this kind of situation, the approach most commonly described in the literature is the closure of the existing fistula. However, the haemodynamic effects in such situations are incompletely understood. In our case, we proceded to revascularisation of the proximal left anterior descendent artery by percutaneous coronary intervention and stent implantation, after which a steal phenomenon was no longer observed and the patient remained asymptomatic. We hence suggest this approach as a possible alternative strategy in patients with internal thoracic artery steal from ipsilateral upper extremity arteriovenous fistula and feasable proximal native vessel revascularisation.

Key words: steal; coronary; bypass; dialysis; fistula; ipsilateral; angina; graft; PCI; percutaneous coronary intervention; stent
\end{abstract}

\section{Case report}

In patients undergoing chronic dialysis through an upper extremity arteriovenous (AV) fistula, the haemodynamic effects on the ipsilateral internal thoracic artery (ITA) are incompletely understood. Reports exist on the occurence of a coronary steal phenomenon in this situation, if the ipsilateral ITA is used as a coronary artery bypass graft (CABG) [1]. However, evidence regarding this matter is conflicting

Funding / potential competing interests: No financial support and no other potential conflict of interest relevant to this article were reported. [2].

We report the case of a 69 -year old woman, undergoing chronic haemodialysis, who was admitted to our hospital due to typical angina pectoris and transitory ST segment elevation in two precordial leads (V1-V2), with angiographic evidence of coronary steal phenomenon at rest.

Six years earlier, she had undergone four-vessel CABG, in which the left ITA was grafted to the mid left anterior descending (LAD) artery, and a sequential saphenous vein graft was attached to the obtuse marginal branch, with jumps to the right posterolateral branch and posterior descending artery. One month af-

Figure 1

Reverse flow in the left ITA bypass, visualised at the first selective injection into the LM coronary artery; the black arrow indicates the stenosis (A). Visualisation of the complete left ITA bypass graft from its anastomosis with the mid LAD coronary artery to the left subclavian artery, after selective injection into the LM coronary artery (B)

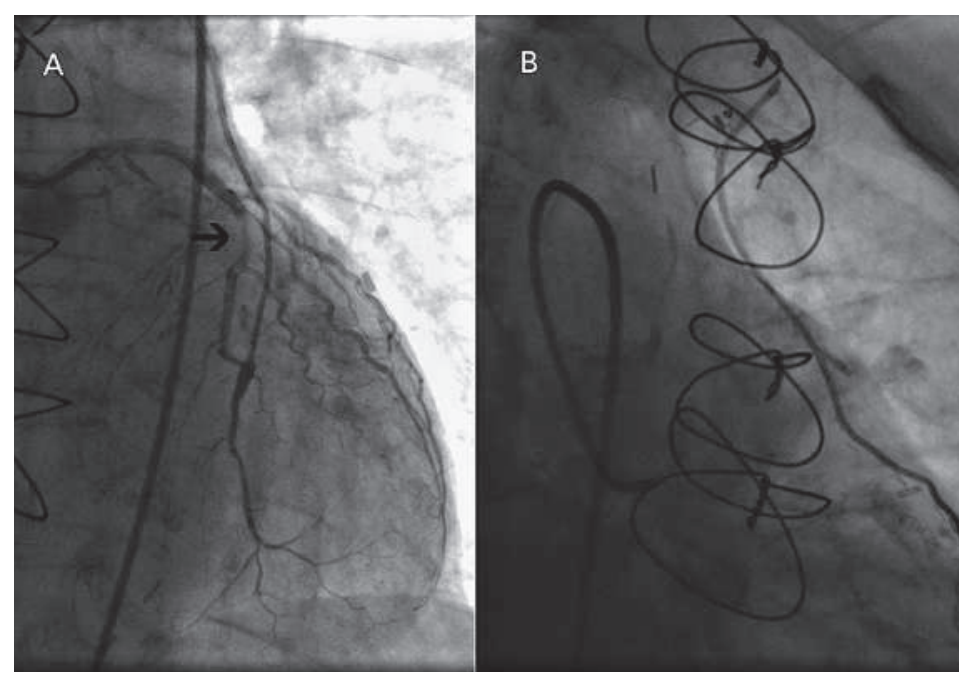

Correspondence:

Sergio Musto D'Amore, MD

Cardiocentro Ticino

Via Tesserete 48

$\mathrm{CH}-6900$ Lugano

Switzerland

sergio.mustodamore[at]cardiocentro.org 


\section{Figure 2}

Demonstration of ITA steal due to AV shunt using a pneumatic cuff to occlude the fistula. Deflated cuff: selective injection into the left ITA graft shows an opposition to antegrade flow within the ITA graft (A and B), and a prominent wash-out of contrast medium through the subclavian vein in the late phase (B). Inflated cuff: normal antegrade flow in the ITA graft (C, D), and absence of wash-out of contrast medium through the subclavian vein (D). Black arrows indicate the maximum range of antegrade bypass flow. Black stars indicate the subclavian vein.

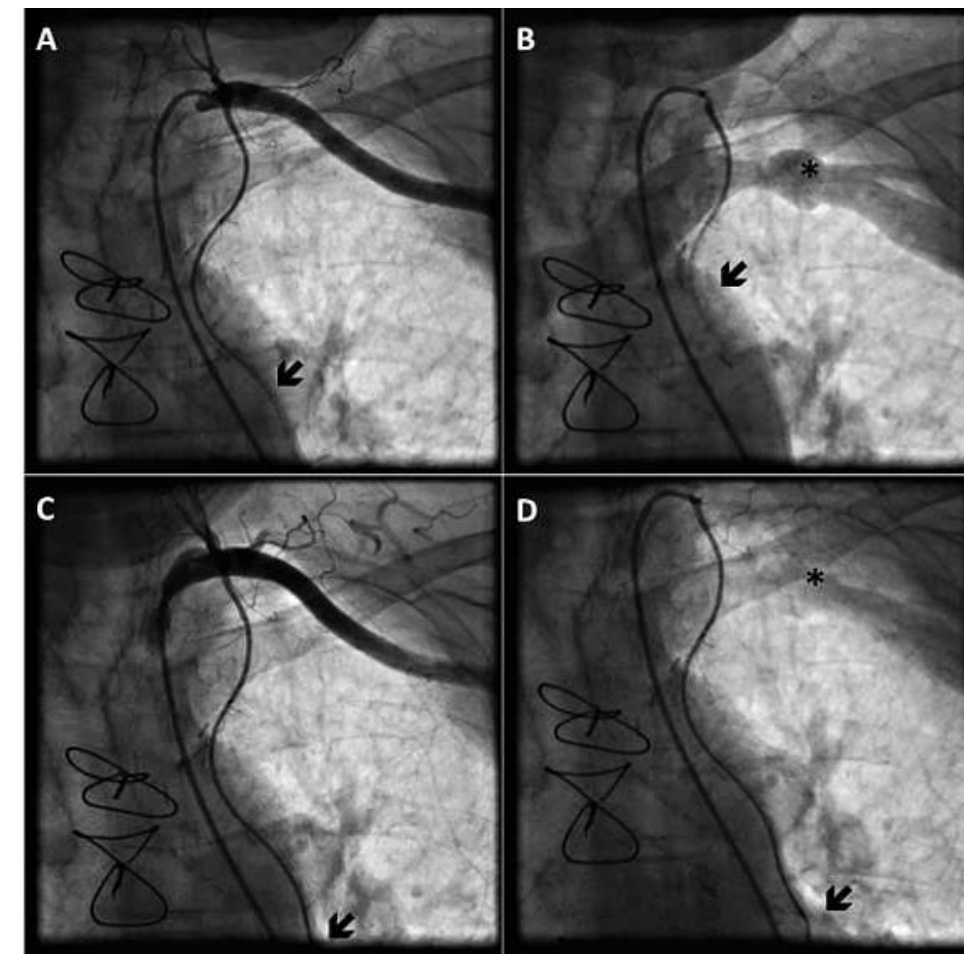

ter CABG surgery, due to renal failure requiring haemodialysis, a left brachiocephalic AV fistula was created. Subsequent dialysis records documented a shunt flow of $350 \mathrm{ml} / \mathrm{min}$.

At the time of hospital re-admission for angina, she had been asymptomatic for six years.

Coronary angiography showed no significant changes compared to the previous exam; all bypass grafts were patent. However, injection of contrast medium into the left main (LM) coronary artery revealed a reverse flow of blood in the left ITA throughout the cardiac cycle, consistent with a coronary steal phenomenon (fig. 1). Even at selective injection into the left ITA, an anomalously competing flow was observed in the bypass graft (fig. $2 \mathrm{~A}$ and $2 \mathrm{~B}$ ). Of note, no pressure damping occurred at the intubation of the bypass graft, which rendered the presence of a significant ostial narrowing unlikely.

We confirmed the presence of steal due to the ipsilateral AV fistula by occluding the shunt with a pneumatic cuff, which resulted in restoration of antegrade bypass flow during both systole and diastole (fig. 2).

In lack of evidence about the best treatment strategy in this situation, we proceeded to revascularisation of the proximal LAD by percutaneous coronary intervention (PCI) and stent implantation, which limited retrograde flow in the graft; a diastolic steal phenomenon was no longer observed (fig. 3 and 4). Nine months later, the patient had not experienced any further anginal symptoms, neither at rest nor during haemodialysis.

\section{Discussion}

In patients undergoing chronic haemodialysis through an upper extremity AV fistula, coronary steal can occur if the patient is subjected to CABG using the ipsilateral ITA [1]. Current practice in dialysed patients undergoing CABG surgery, is to use the ITA on the opposite side of the shunt, or a free ITA graft, if preoperative angiography reveals a retrograde diastolic flow (i.e., steal) in the ipsilateral ITA [3]. Only a few reports and small studies have examined the interference between upper extremity AV fistulae and ipsilateral ITA used as coronary grafts, with controversial results [1, 2].

\section{Figure 3}

Demonstration of blood flow in the ITA bypass graft before (upper side) and after (lower side) PCI. The arrows indicate the retrograde extension of contrast media in the bypass graft during injection into the LM coronary artery. Corresponding arrows are represented in the white panel to the right, indicating the extension of contrast media in the ITA (red line) in systole (S) and diastole (D) with respect to the point of anastomosis (black horizontal line).

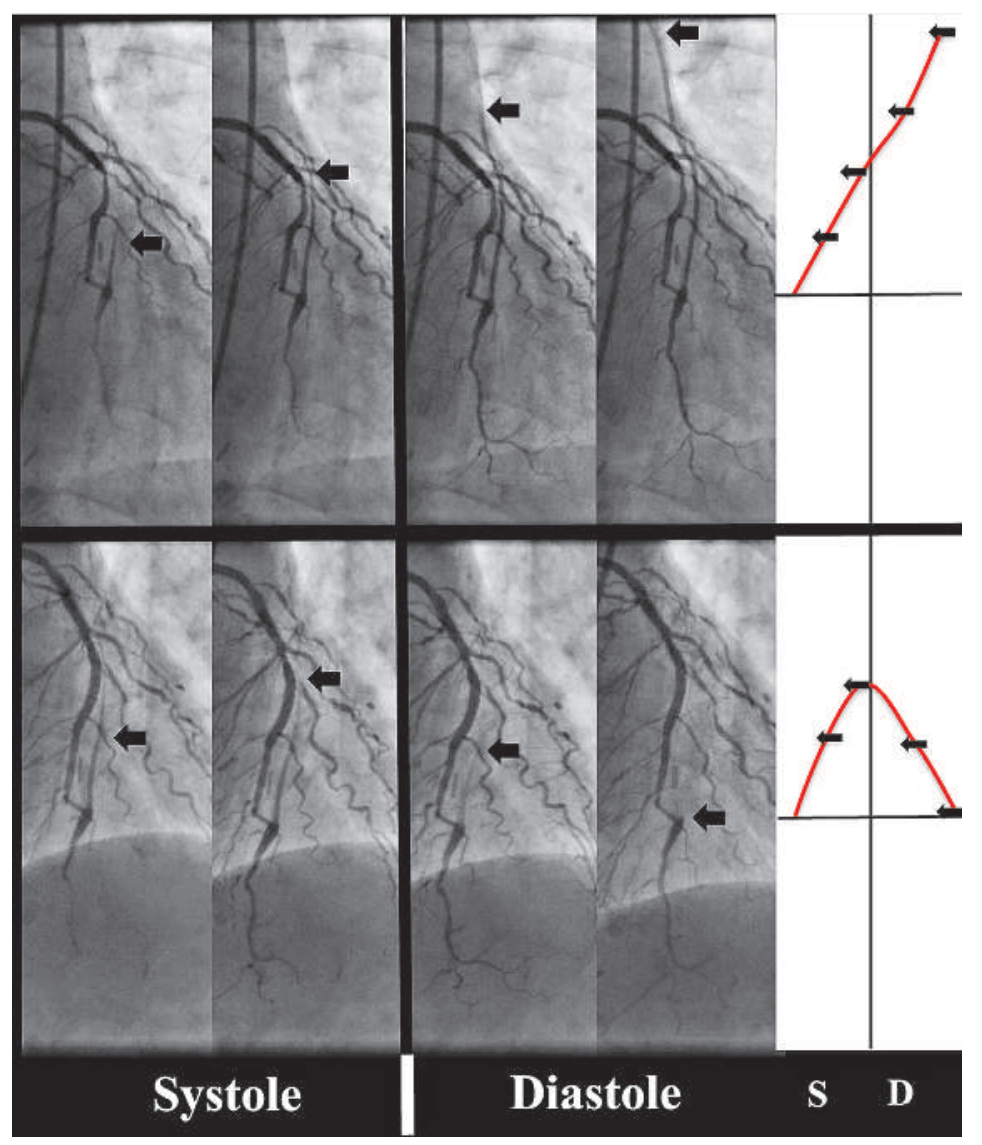


Figure 4

After stent implantation (black arrow), retrograde flow of contrast medium in ventricular systole is limited.

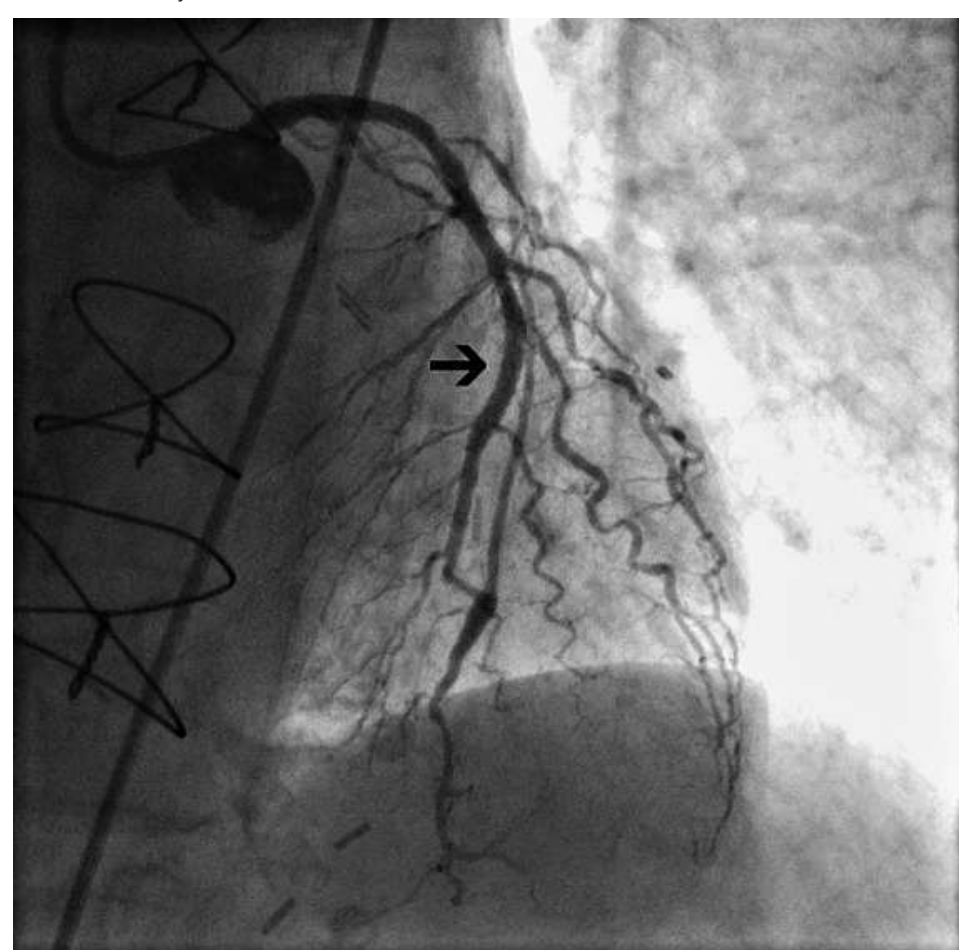

Based on the data currently available it seems impossible to predict coronary steal in this patient group. It is also unclear to what extent the amount of shunt flow contributes to ipsilateral ITA steal in patients who are on dialysis [3]. In symptomatic cases, closure of the existing fistula has been suggested as an effective treatment strategy [4].
Our decision to revascularise the native vessel was driven by the intent of rendering it independent from a functionally defective bypass. The reduction of steal subsequently observed, seems to derive from an important reduction of coronary artery resistance after stenting the proximal portion of the vessel, leading to a crucial increase in coronary reserve.

Pathophysiologically, a brachiocephalic AV fistula creates a low resistance zone in the entire region of the ipsilateral subclavian artery and its periphery. It is conceivable that this effect can be transmitted through the ipsilateral ITA [1], creating a sucking effect on the bypassed vessel. We hence postulate having achieved a cease of steal from the native LAD by restoring its coronary reserve, reducing its intrinsic resistance to flow.

We propose this approach as a possible alternative strategy in patients with internal thoracic artery steal from ipsilateral upper extremity arteriovenous fistula and feasable proximal native vessel revascularisation.

\section{References}

1 Gaudino M, Serricchio M, Luciani N, Giungi S, Salica A, Pola R, et al. Risks of using internal thoracic artery grafts in patients in chronic hemodialysis via upper extremity arteriovenous fistula. Circulation. 2003;107(21):2653-5.

2 Rahbar R, McGee WR, Birdas TJ, Muluk S, Magovern J, Maher T. Upper extremity arteriovenous fistulas induce modest hemodynamic effect on the in situ internal thoracic artery. Ann Thorac Surg. 2006;81(1):145-7.

3 Kato H, Ikawa S, Hayashi A, Yokoyama K. Internal mammary artery steal in a dialysis patient. Ann Thorac Surg. 2003;75(1):270-1.

4 Ginzburg V, Margulis G, Greenberg G, Mayzler O, Wolak T, Tovbin D, et al. Preserving the left arm vein in cases of hemodialysis access generating left internal mammary artery steal syndrome. J Vasc Access. 2004;5(3):133-5. 